\title{
Structure of laponite-styrene precursor dispersions for production of advanced polymer-clay nanocomposites
}

\author{
R. Fartaria, ${ }^{a}$ N. Javid, ${ }^{a}$ R. A. Pethrick, ${ }^{b}$ J. J. Liggat, ${ }^{b}$ J. Sefcik ${ }^{a}$ and M. B. Sweatman $* a$ \\ Received 19th May 2011, Accepted 18th July 2011 \\ DOI: $10.1039 / \mathrm{c} 1 \mathrm{sm05935a}$
}

One method for production of polymer-clay nanocomposites involves dispersal of surface-modified clay in a polymerisable monomeric solvent, followed by fast in situ polymerisation. In order to tailor the properties of the final material we aim to control the dispersion state of the clay in the precursor solvent. Here, we study dispersions of surface-modified Laponite, a synthetic clay, in styrene via large-scale Monte-Carlo simulations and experimentally, using small angle X-ray and static light scattering. By tuning the effective interaction between simulated laponite particles we are able to reproduce the experimental scattering intensity patterns for this system, with good accuracy over a wide range of length scales. However, this agreement could only be obtained by introducing a permanent electrostatic dipole moment into the plane of each Laponite particle, which we explain in terms of the distribution of substituted metal atoms within each Laponite particle. This suggests that Laponite dispersions, and perhaps other clay suspensions, should display some of the structural characteristics of dipolar fluids. Our simulated structures show aggregation regimes ranging from networks of long chains to dense clusters of Laponite particles, and we also obtain some intriguing 'globular' clusters, similar to capsids. We see no indication of any 'house-of-cards' structures. The simulation that most closely matches experimental results indicates that gel-like networks are obtained in Laponite dispersions, which however appear optically clear and non-sedimenting over extended periods of time. This suggests it could be difficult to obtain truly isotropic equilibrium dispersion as a starting point for synthesis of advanced polymer-clay nanocomposites with controlled structures.

\section{Introduction}

Polymer-clay nanocomposites, formed by dispersing suitably modified clay within polymer, are known to impart enhanced properties ${ }^{1,2}$ such as mechanical strength, gas impermeability and fire retardance, to polymers. However, current methods for producing these materials lack fine control over the dispersed state of clay platelets, and so the resulting materials are not optimal. Achieving fine control over the platelet dispersion state would yield the next generation of nanocomposite materials with novel and further improved properties. Unfortunately, even the most advanced of current synthesis methods, ${ }^{3}$ which are generally based on mechanical mixing of clay and a polymer melt, lack the fine control needed to produce optimised materials. Although reasonable alignment, exfoliation (i.e. delamination), and dispersion can be achieved at low loadings for some polymer-clay combinations, ${ }^{2}$ at higher loadings this melt-mixing method leads to poorly aligned, exfoliated and dispersed platelets. Moreover,

${ }^{a}$ Department of Chemical and Process Engineering, University of Strathclyde, Glasgow, G1 1XJ, Scotland, UK

${ }^{b}$ Department of Pure and Applied Chemistry, University of Strathclyde, Glasgow, G1 $1 X L$, Scotland, UK this method cannot be used with polymers that degrade at melt temperatures. Of all production methods, in situ polymerisation has the greatest potential for generating optimal materials, since in principle the state of the platelet dispersion can be controlled by thermodynamic equilibrium in the monomer liquid state (this is not possible in a polymer melt) and then 'locked-in' by fast in situ polymerisation. The production polymer-clay nanocomposites, with fully exfoliated platelets, has been reported in several works. ${ }^{4-6}$ Unfortunately, isotropic dispersion of clay in polymerisable solvents has never been demonstrated, although recent work suggests that it might be possible if clay particles are suitably modified. For example, work by Leach et al. ${ }^{7}$ suggests that Montmorillonite (MMT) and Laponite clays (MMT is a natural smectite clay while Laponite is a synthetic hectorite clay) can be dispersed in toluene if they are suitably sterically stabilised. However, their work did not assess the long-range structure of their dispersions, so it is not clear whether these were actually isotropic dispersions of disconnected (non-networked) particles or particles aggregated into clusters or gels. Also, work by some of us (RP and JL) ${ }^{8}$ suggests that MMT could be dispersed in methyl methacrylate if it is treated with LICA-44, a titanate surface coupling agent. Here, MMT is exfoliated via sonication and the viscosity of the resulting dispersion is 
significantly reduced on addition of LICA-44, without apparently affecting the degree of exfoliation, indicating a significant reduction in the degree of any clustering or networking.

These studies represent the first tentative steps towards novel next-generation polymer-clay nanocomposites and applications. On the other hand, Pluart et.al. ${ }^{9}$ found, on the basis of rheological studies, that MMT dispersed in epoxy and amine solvents forms different kinds of gels depending on the effective interactions between clay particles, which depend both on the type of organic solvent and stabilising surfactant. They explain differences in observed viscosity and shear thinning behaviour in terms of these different 'weak' and 'strong' gel structures. Weak gels have a much lower yield stress than strong gels, exhibit much more pronounced shear thinning behaviour, and reform relatively quickly. Strong gels, on the other hand, display nearly Newtonian flow behaviour once the gel is broken, and do not reform (or are slow to reform) when quiescent. At a microscopic level this is interpreted in terms of different kinds of platelet structures and therefore effective platelet interactions. Weak gels are thought to consist of more open and diffuse networks caused by weaker effective interparticle interactions and therefore form better dispersions, while strong gels are formed from more compact networked strands caused by stronger effective interparticle interactions. Also, work involving environmental SEM imaging and rheological studies by Oberhauser et al. ${ }^{10}$ indicates that MMT, even with an excess of stabilising surfactant, forms gels in p-xylene. Note that non-equilibrium gels generally represent an uncontrolled dispersion state, and so are undesirable in the context of this present work. Although the solvents used in the aforementioned work are all different kinds of organic solvent, one might expect to see reasonably similar dispersion behaviour. A potentially more significant difference in these works is the kind of stabilising surfactant used. The use of LICA $-44^{8}$ and the SAP stabiliser ${ }^{7}$ is thought to reduce edge-face interactions and hence lead to improved dispersion. So perhaps elimination of edge-face interactions is the key to control of clay dispersion structure in organic solvents.

The aim of this work is to investigate this question in the context of suitably modified Laponite in styrene to determine whether this system can indeed be used to make the next generation of polymer-clay nanocomposites. We focus on Laponite because its relatively narrow size and shape polydispersity, compared to natural clays like MMT, will allow better comparison between experiments and simulations. Our overall strategy compares experimental static light scattering (SLS) and small angle X-ray scattering (SAXS) investigations of Laponite dispersions with Monte-Carlo simulation results. This particular paper is devoted to description of the simulation work, and its validation by comparison with experimental data, while details of the more wide ranging experimental work will be presented elsewhere. We present our work here as follows. In the next section we briefly describe the experimental system of surface modified Laponite in styrene. We then describe our simulation strategy for modelling this system, our effective interaction models, and details of our simulation methods. Next we describe the results of simulations that attempt to mimic a range of conditions and possibilities for effective Laponite-Laponite interactions. These results are compared with experimental SLS and SAXS results obtained from surface-modified Laponite dispersions, which are used to refine our simulation models. We finally draw conclusions about the nature of (surface-modified) Laponite effective interactions in organic solvents, as well as the kinds of structure found in Laponite dispersions.

\section{Experimental system}

Laponite is a synthetic 2:1 layered phylosilicate of the hectorite family. This means it is formed from a sandwich of two tetrahedral silica layers and a middle layer of octahedrally coordinated magnesium oxide (and hydroxide). The metal layer is highly defective, with many of the magnesium atoms randomly substituted by lithium atoms. This leads to a planar crystal with overall negative charge, which is counterbalanced by surface adsorbed cations, typically sodium. The crystal edges, or rims, will likely consist of a variety of terminal states consistent with an interrupted crystal growth process. The size and shape of Laponite crystals have been previously determined by AFM studies ${ }^{11}$ to be fairly regular, with small polydispersity in thickness and shape. These studies determined that Laponite particles are elliptical in shape with a larger axis of $25 \mathrm{~nm}$ (at the peak of the distribution) and an eccentricity of 0.8 . Thickness was determined to be $1 \mathrm{~nm}$ (at the peak of the distribution). Therefore, for the purposes of the present study, Laponite particles will be considered as having a circular shape, with diameter $D=25 \mathrm{~nm}$ and thickness $L=1 \mathrm{~nm}$. To make the platelets organophillic the sodium cations are exchanged with an organic cationic surfactant. For favourable surfactant-organic solvent interactions it should then be possible to obtain well dispersed states, perhaps via sonication, in organic solvents. However, the crystal edges might still be relatively 'bare' after this treatment, and so further steric stabilisation might be required to obtain good dispersions. The aim of further experimental work carried out in parallel with this work is to ascertain the effectiveness of different surface treatments for complete isotropic dispersal of Laponite in styrene.

In our room temperature experimental work structural characteristics of dispersed Laponite are determined by a combination of small-angle X-ray scattering (SAXS) and static light scattering (SLS). We tested many different kinds of Laponite surface treatment and preparation methods, but we found that Laponite formed particle clusters or networks in styrene at all platelet concentrations regardless of our efforts. Complete details of the extensive experimental methods and results will be presented in a following paper. Here we instead focus on the best result we obtained, in the sense that it generated the most open particle network (as measured in terms of the exponent in the power law scaling of the SAXS and SLS intensity vs. scattering vector amplitude, see Fig. 1), for comparison with our simulation work. This involved a combined treatment consisting of silylation of Laponite, with faces already organically modified with methyl tallow bis-2-hydroxyethyl ammonium cation (cation exchange capacity is 90 mequiv/100 g; supplied by Rockwood Additives Ltd), and then further modification with SAP (polyisobutylene based stabiliser; supplied by Infineum UK). The organically modified Laponite was suspended in ethanol under stirring and phenyl-trimethoxy-silane (supplied by SigmaAldrich) was added to the suspension, which was then stirred and heated at $65^{\circ} \mathrm{C}$ while refluxing for $2 \mathrm{~h}$. The resulting suspension 


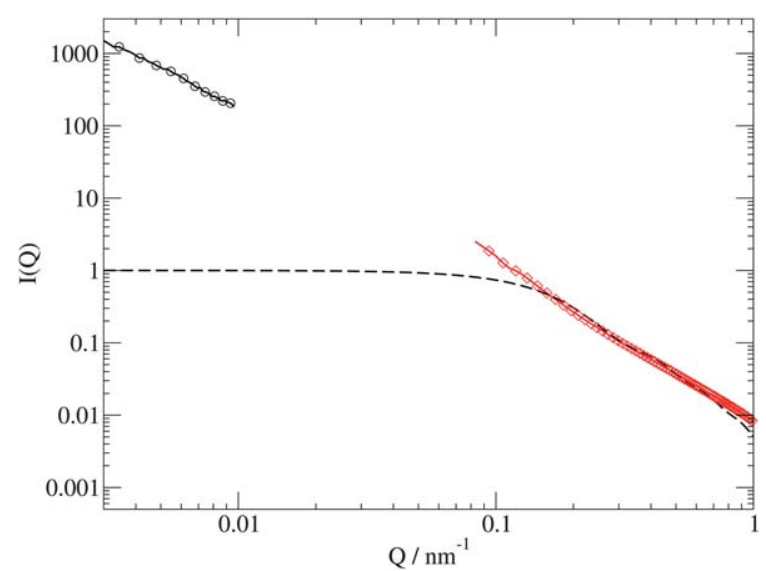

Fig. 1 Scattering intensities (in a.u.) obtained by SAXS (red diamonds) and SLS (black circles) for a suspension of $1 \%(\mathrm{w} / \mathrm{w})$ organically modified Laponite in styrene. The black dashed line corresponds to the form factor of a single platelet with a diameter $D=27 \mathrm{~nm}$ and thickness $L=3 \mathrm{~nm}$.

was filtered and washed with ethanol to remove the excess silane and solids were filtered and dried at $65^{\circ} \mathrm{C}$. The silylated Laponite was dispersed in styrene with added 1 wt $\%$ of SAP by stirring sonication method. Our observation that SAP is a good stabilisation agent agrees with the findings of Leach et al. ${ }^{7} \mathrm{We}$ expect this combined treatment to be more effective than others because of the way that it stabilises platelet edges. Silylation in particular is designed to reduce the potential for edge interactions.

For comparison with the computational study we used a $1 \%(w / w)$ system consisting of the above surface modified Laponite dispersed in styrene. These suspensions are apparently homogeneous, stable (within a time frame of a few hours), with very low viscosity when compared to other samples produced with other surface modification strategies. Synchrotron small angle X-ray scattering measurements were carried out at beamline BL9 of DELTA synchrotron source in Dortmund, Germany and at beamline A2 in Hasylab at DESY synchrotron source Hamburg, Germany. The CCD or image plate scattered intensity patterns were integrated by programme FIT2D to obtain the 2D scattering data. Background intensities were subtracted from the measured intensities as appropriate. Static light scattering experiments were carried out by using the 3DDLS spectrometer (LS instruments, Fribourg, Switzerland) using vertically polarized He-Ne laser light (wavelength of $632.8 \mathrm{~nm}$ ) with an avalanche photodiode detector. Scattered intensities were recorded for scattering angles between 15 to 135 degrees and background intensities were subtracted from the measured intensities as appropriate. All measurements were carried out at $25^{\circ} \mathrm{C}$.

We will briefly analyse the scattering intensities, presented on Fig. 1, as they form the basis for development of the computational model. The abscissa of this plot refers to the scattering vector amplitude $Q=(4 \pi / \lambda) \sin (\theta)$, where $2 \theta$ is the scattering angle, ${ }^{12}$ which is related to the range, $d$, of structural correlations in the system by the expression $Q=2 \pi / d$. The results from SAXS show a power law scaling with an exponent of -2 , with absence of any peaks, indicating that the Laponite platelets in this system are not stacked or otherwise correlated at lengthscales smaller than their diameter. However, in a fully dispersed isotropic system without any particle clustering one would expect to see the intensity plot (in $\log / \log$ scale) plateau at lower $Q$ values corresponding to the Guinier region for individual platelets. So, for a fully dispersed system, we would expect to see a completely flat profile for the $Q$ region probed by our SLS measurements due to the size of Laponite particles, as can be seen from theoretical scattering expected from individual laponite particles as shown by form factor $P(Q)$ in Fig. 1. In our system, though, we observe that the power law plot, starting in the SAXS range, extends to very low $Q$ values indicating the presence of very large structures. Furthermore, the slope of the intensity plot in the SLS region, $-1.8 \pm 0.02$, with no peaks, is consistent with the formation of very low density structures, such as fractal-like clusters or networks of particles. ${ }^{13}$ From these experimental results alone it is not possible to fully characterise the structure of the clusters or networks that were formed in this and similar systems we also prepared. The aim of the computational study, presented below, is to provide additional insight into the nature of these experimental structures and the effective interactions between individual Laponite platelets, and hence to suggest suitable conditions and surface modifications for the formation of good isotropic dispersions.

\section{Simulation details}

We wish to simulate surface modified Laponite in styrene over a wide range of length scales relevant to SAXS and SLS. To achieve this we adopt a strategy based on integrating out the solvent degrees of freedom. To justify this strategy consider that each Laponite particle in solution will perturb the solvent in a region surrounding it out to a range, typically, of a few solvent molecules, and the magnitude of this perturbation will decay rapidly with distance. Therefore, the zone of interference between these perturbed regions when two Laponite particles approach each other is generally relatively small, compared to Laponite particle sizes, unless the Laponite particles happen to be parallel and relatively close. Because of specific electrostatic interactions between Laponite particles, we expect such parallel configurations are highly unlikely. Moreover, for the same reasons we consider higher order (three-body etc) solvent perturbation effects to be even less significant. It follows that the contribution of solvent perturbations to the effective interaction between Laponite particles can be neglected, especially compared to direct Laponite-Laponite interactions of electrostatic origin. These arguments can been expressed more formally using the theoretical treatment of Dijkstra et al. ${ }^{14}$ On this basis, we expect Laponite dispersions can be modelled adequately in terms of effective pair interactions that ignore solvent perturbations. The most significant of these are i) hard-core (excluded volume) interactions that prevent particle overlaps, and ii) electrostatic interactions. Dispersion (London) effective platelet-platelet pair interactions, being shorter in range, are considered less significant than electrostatic interactions and we also neglect them here.

\subsection{Models for Laponite in styrene}

For convenience we model the excluded volume interactions of surface modified Laponite particles in terms of the hard cutsphere model. ${ }^{15,16}$ In reality Laponite particles are not quite 
circular (although they are relatively thin and flat), and we expect the surfactant layers on particle surfaces to exhibit a mutual soft repulsion rather than a hard-core interaction. But we do not expect these differences to be significant, and so we consider our model to be adequate. Actually, we consider our platelet model to comprise two concentric cut-spheres (see Fig. 2a): an inner one with thickness $L=1 \mathrm{~nm}$ and diameter $D=25 \mathrm{~nm}$ at the centre of an outer one with thickness and diameter increased by a certain distance, $S$, beyond the inner one. The inner cut-sphere represents the Laponite crystal and the outer one represents the surfactant layer covering the platelet. Laponite crystals bear negative charge (nearly $-700 \mathrm{e}$ ) on each face, and positive charge (nearly $+70 e$ ) on their rims. ${ }^{17-23}$ The negative charge on the faces is compensated by a layer of adsorbed cations, either $\mathrm{Na}^{+}$or cationic surfactant. This charge distribution is represented in our model by four layers of charge, each bearing 700 interaction sites distributed in a uniform pattern, located at the surface of the inner cut-sphere. The pattern for the distribution of charge is shown in Fig. $2 b$ and the charge layers are diagrammatically represented on Fig. 2c. The two inner layers are negative and the two outer layers are positive and bear half of the face charge. Analogously, two charged rings are placed at the rims of the inner cut-sphere, as shown in Fig. 2b. The inner rim is positively charged with a total charge of $-70 e$ and the outer rim is negatively charged by the same amount. Positive and negative charges, in faces and rims, are separated by a distance of $2.4 \AA$. We assume, then, that in the same way that the faces have a layer of adsorbed counterions, the rims are also covered by a layer of anions in order to maintain electroneutrality. This distribution of platelet charge exhibits a quadrupole moment perpendicular to the faces, and a null dipole moment, and is comparable at large separations to other quadrupolar models used in the literature $^{24-27}$ for the simulation of aqueous dispersions of Laponite. We will refer to this basic model as "quadrupole plus repulsive surfactant" (QPRS). One simple way of approximating the interaction energy would be to use a point quadrupole at the centre of the platelets but this would be accurate only at large distances. ${ }^{28}$ At short distances it would introduce spurious effects returning incorrect energies for some configurations that would have to be corrected. ${ }^{17,18}$

Using a large number of sites for the placement of partial charges enables a more accurate description of the electrostatic interactions between platelets but on the other hand it becomes prohibitively expensive to calculate the interaction energy for the large number of platelets required in a simulation. It is therefore necessary to tabulate the interaction for a pair of platelets. To that effect, the following coordinate system (see Fig. 3) is used to identify the configuration between two platelets: the distance between the geometric centres of the platelets, $r$, the angle between the vector (r) connecting the geometric centres and the vector perpendicular to the faces of the first platelet $\left(\mathbf{n}_{1}\right), \theta$, the angle between the vector perpendicular to the faces of the second platelet $\left(\mathbf{n}_{2}\right)$ and $\mathbf{n}_{1}, \gamma$, and the angle between the projection of the vectors $\mathbf{r}$ and $\mathbf{n}_{2}$ on the equatorial plane of the first platelet, $\phi$. The interactions are tabulated, covering all the space up to a sphere of radius of $75 \mathrm{~nm}$ at which distance the energy values are already negligible (less than $1 \%$ of the minimum of the interaction at short distances). The sampling intervals used in the construction of the table are $\Delta r=0.2 \mathrm{~nm}$
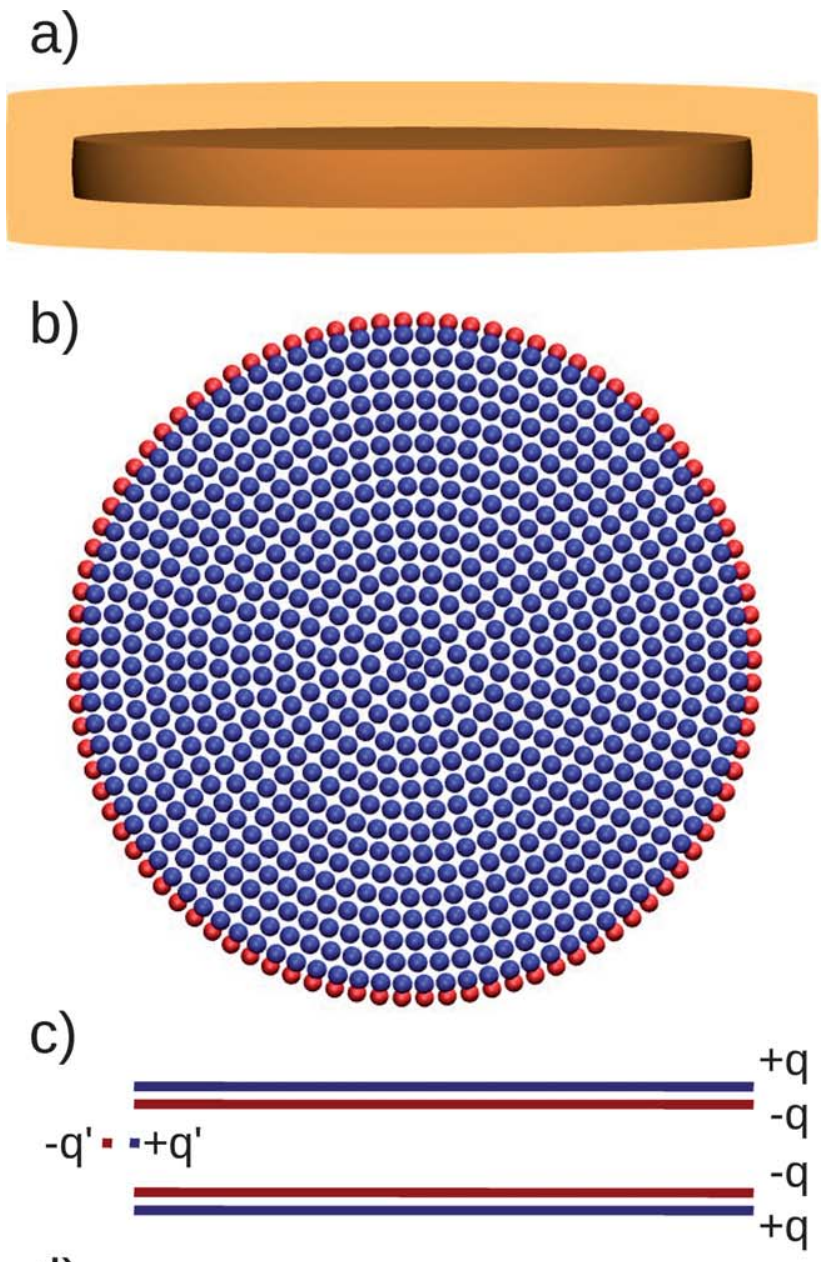

d)
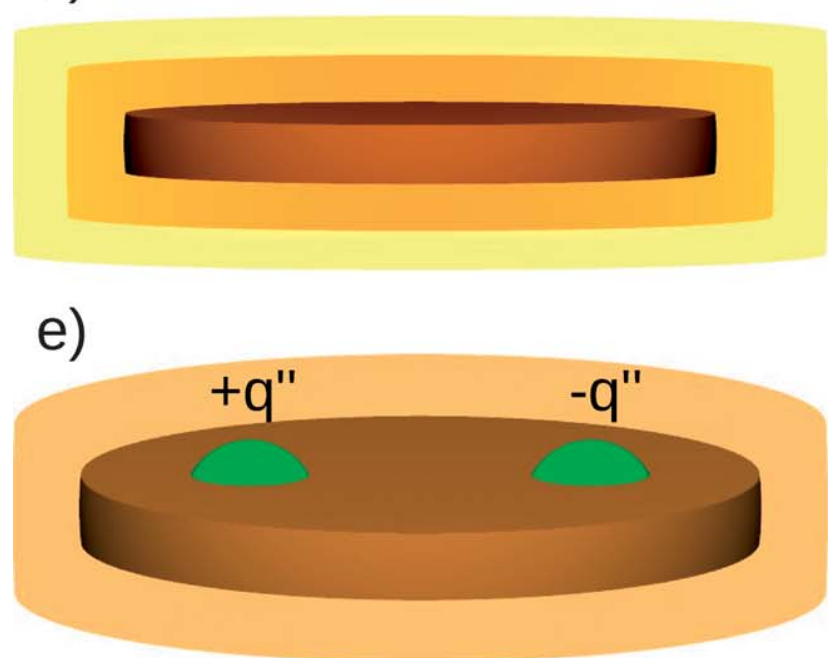

Fig. 2 a) Double cut-sphere model: the inner disk represents the Laponite crystal and the outer disk represents the organic modification. b) Charge pattern used to cover the surface (blue) and rim (red) of the Laponite crystal. c) Diagram showing the charge layers used on the faces and $\operatorname{rim}\left(q\right.$ and $q^{\prime}$ are, respectively, the surface and rim charge densities). d) Triple cut-sphere model: a layer of attractive surfactant, represented by the yellow disk, is added to the model in a). e) Double cut-sphere model incorporating a dipole, simulated by the two charges, $q^{\prime \prime}$. 
and $\Delta \theta=\Delta \gamma=\Delta \phi=\pi / 40 \mathrm{rad}$. In the simulations the electrostatic contribution to the energy from a pair of platelets is calculated from the table by a tetra-linear interpolation. ${ }^{29}$ Coulomb interaction energy curves for a group of selected orientations, $\{\theta, \gamma, \phi\}$, are presented in Fig. 4. As expected, from what is effectively a quadrupole-quadrupole interaction at large separations, the configurations for parallel plates, $\{0,0,0\}$, are always repulsive (which supports our argument above about neglecting solvent perturbation effects) and the T-configuration, $\{0,90,0\}$, is attractive. But unlike a quadrupole-quadrupole interaction, the configurations that are most attractive, within the limits imposed by hard disk repulsion, are when the angles between the normals to the faces are of about 20 degrees and the platelets approach each other along the equatorial plane. This behaviour cannot be reproduced with simple point multipole interactions. We initially tried several different interaction models for electrostatics, including a point quadrupole model and models that used fewer partial charge sites. But we found that these models could not reproduce the same qualitative behaviour obtained with the full resolution model used here. A similar account of the problems encountered in using lower resolution electrostatic interaction models for platelet systems is given by Odriozola et al. ${ }^{30}$

In order to understand the influence of the surfactant layer on platelet interactions and dispersion structure two modifications to the previous model are tested. The first involves using a different thickness of surfactant repulsive layer. This truncates the electrostatics at different ranges making them softer for larger surfactant thickness (see Fig. 4). The second is to add a third cutsphere to the model (see Fig. 2d) representing a layer of attractive surfactant. This tests the hypothesis that there could be some

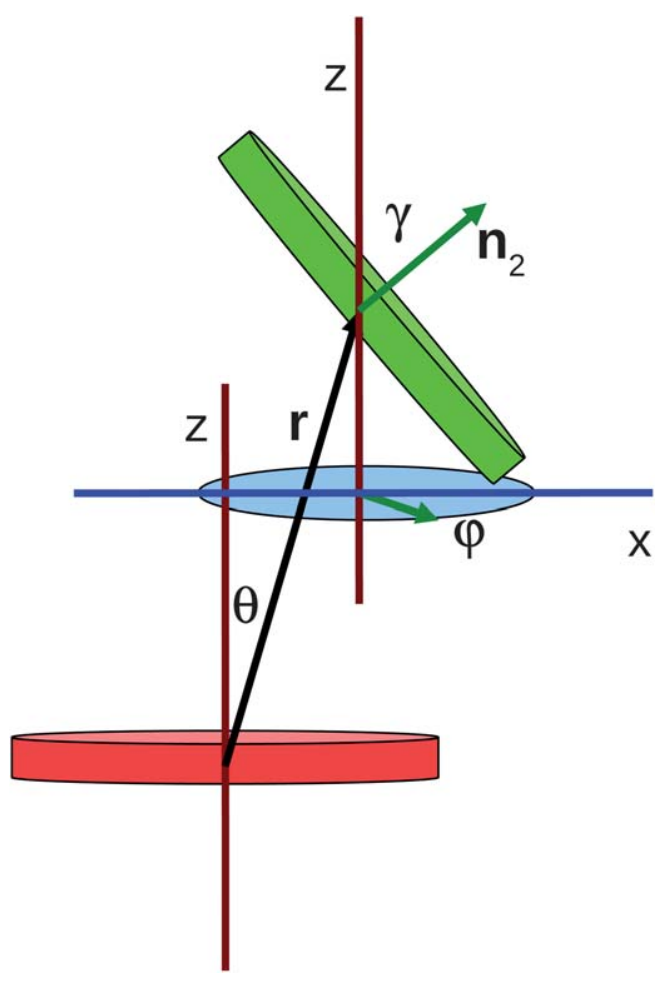

Fig. 3 Coordinate system used to identify the configuration between two platelets. Symbols are identified on the text. effective attraction between platelets caused by surfactant, either by attractive effective interactions between surfactant tails, or perhaps due to bridging of surfactant between platelets.

As a final modification we also test the possibility that there might be a permanent electrostatic dipole in the plane of the Laponite platelet. Although the origin of any electrostatic dipole is not obvious, we suggest that it is unlikely that any particular platelet will have a perfectly symmetric distribution of substituted metal atoms or terminating atoms around its edge. Therefore, each Laponite disk might be expected to exhibit a residual electrostatic dipole in the plane of the crystal. Although any such electrostatic dipole would be counterbalanced, or screened, to some degree by relatively mobile cationic surface charges $\left(\mathrm{Na}^{+}\right.$or cationic surfactant), these residual dipole - dipole interactions might still be significant at small pair separations. So, an in-plane electrostatic dipole is added by placing two additional charges symmetrically along the platelet's diameter (see Fig. 2e) at a distance from each other of two thirds of the platelet diameter. Dipole-dipole interactions are calculated with the screened potential

$$
U_{i j}=U_{\text {dipole }} \times \exp \left(-r_{i j} / \lambda_{D}\right)
$$

where $U_{\text {dipole }}$ is the standard Coulomb energy of the four chargecharge interactions comprising the dipole and $\lambda_{D}$ is the characteristic Debye screening length ${ }^{31}$ which, in our case, is set to six platelet diameters. A fortunate consequence of screening the dipole potential in our calculations is that it speeds up simulations by avoiding more computationally expensive methods, e.g. Ewald summation. Ideally, one would like to use a larger number of partial charges to represent this dipolar interaction, as for the faces and rims. But, unfortunately, the inclusion of a dipole breaks the symmetry of the charge distribution in the platelets, and the additional degrees of freedom would render tabulation of the pair-interaction too expensive (the required table would need gigabytes of memory to be stored). For the same reason, we do not include the interaction between the screened dipole and the original charge distribution of the QPRS model. We expect this

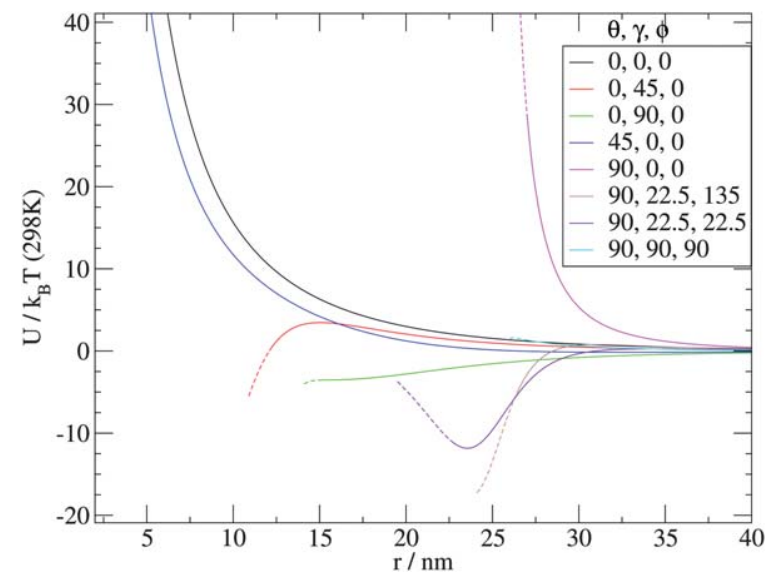

Fig. 4 Electrostatic potential energy, $U$, for the QPRS model. A representative set of relative orientations of platelets is shown. Dashed lines represent the continuation of the interaction for distances corresponding to surfactant overlap. 
simplified platelet dipole model to be sufficient to capture the main features of this dipole - dipole interaction.

\subsection{Monte Carlo simulations}

Monte Carlo simulations are performed using the NVT ensemble (constant number of particles $N$, constant volume $V$, and constant temperature $T$ ) with number density $2.302 \times 10^{-6} \mathrm{~nm}^{-3}$ and $N=7776$ platelets. This density corresponds approximately to a volume fraction of $\phi=0.0035$ and approximately $0.5 \%$ $(w / w)$ of organically modified Laponite in styrene which belongs to the interval of densities studied experimentally. Each simulation ran for more than $10^{6}$ Monte Carlo cycles where each cycle corresponds to, on average, a translation and a rotation of each particle in the system. Standard cluster moves ${ }^{32}$ are used in order to improve the sampling of networked structures. Cluster bonds are constructed for pairs of particles where the distance between their centres of mass is less than $1.1 \mathrm{D}$, and clusters are built for sets of particles mutually connected by any possible bonded chain of particles. After building a set of clusters, a number of translation and rotation moves are tried with the appropriate acceptance probabilities. ${ }^{32}$ A new set of clusters was built, on average, every 100 Monte Carlo cycles. Solvent dispersion interactions are ignored in these simulations but the dielectric constant ( $\varepsilon \sim 2.4$ for styrene) is included. The initial configuration for each simulation is a fully dispersed suspension obtained by simulating the system at high temperature. During simulations evolution of the radial distribution function (RDF) is monitored and analysed by block sampling. For cases where the system forms arrested cluster or networked structures the RDF and energy of the system evolve very slowly as any significant changes in the system can only be achieved by concerted individual movements of the platelets (because cluster moves are very rare). In this case, the last set of RDFs is used to calculate structure factors and scattering intensities of those networks. Simulated RDFs are always converted to scattering intensities because characteristics of both short (corresponding to SAXS) and long-range (corresponding to SLS) structural correlations important in this study are most readily seen in the latter plots, as discussed in the previous section. The normalised scattering intensity is given by

$$
I(Q)=S(Q) P(Q)
$$

where $I$ is the scattering intensity, $S$ is the static structure factor, $P$ is the form factor, and $Q$ is the scattering vector amplitude. The form factor is the scattering intensity from a single platelet and is given by ${ }^{12,33}$

$$
\begin{gathered}
P(Q)=C \nu^{2} \int_{0}^{\pi / 2}\left\{\frac{\sin [Q L \cos (\beta) / 2]}{Q L \cos (\beta) / 2}\right\}^{2} \\
\left\{\frac{2 \mathbf{J}_{1}[Q D \sin (\beta) / 2]}{Q D \sin (\beta) / 2}\right\}^{2} \sin (\beta) d \beta
\end{gathered}
$$

where $C$ is a constant related to the scattering properties of the platelets and the medium, $\nu$ is the volume of one platelet (e.g. cutsphere), $\mathbf{J}_{1}$ is the first order Bessel function and $\beta$ is the angle between the platelet axis (director vector) and the scattering vector, $\mathbf{Q}$. The static structure factor, $S$, is related to the radial distribution function by a sine Fourier transform ${ }^{12,34}$

$$
S(Q)=1+4 \pi \rho Q^{-1} \int_{0}^{\infty}[g(r)-1] r \sin (Q r) d r
$$

where $\rho$ is the number density. Each scattering intensity plot, both the experimental and simulation ones, can be arbitrarily shifted vertically since $C$ is different for the SAXS and SLS experiments. So we have made a judgement about how to place these intensity plots. This adjustment is allowed because results are analysed primarily in terms of the slopes of the plots in each region. Because we can only calculate the RDF up to half of the simulation box length the minimum wave vector is $\lambda=2 \pi /(L / 2)=0.00803$.

\section{Simulation results}

\subsection{Model with quadrupole plus repulsive surfactant}

Above a certain, system dependent, crossover temperature all the systems form stable isotropic dispersions showing no long-range orientational correlations or clustering. For the "quadrupole plus repulsive surfactant" (QPRS) model the reduced crossover temperature is about 0.045 . Throughout this work, temperature will be presented in reduced units using the standard formula, $T^{*}=T k_{B} / \varepsilon$, where $\varepsilon$ is the minimum interaction energy for the QPRS model, $\varepsilon=0.437 \mathrm{eV}$ (see Fig. 4). As can be seen in Fig. 5, the simulated scattering intensity shows the expected plateau for $Q<0.1 \mathrm{~nm}^{-1}$ at temperatures above the crossover temperature. For lower temperatures the system is not ergodic any more and fails to reach equilibrium, and either forms low density networked structures, that percolate the whole system, or a dispersion of clusters that very slowly incorporate the remaining
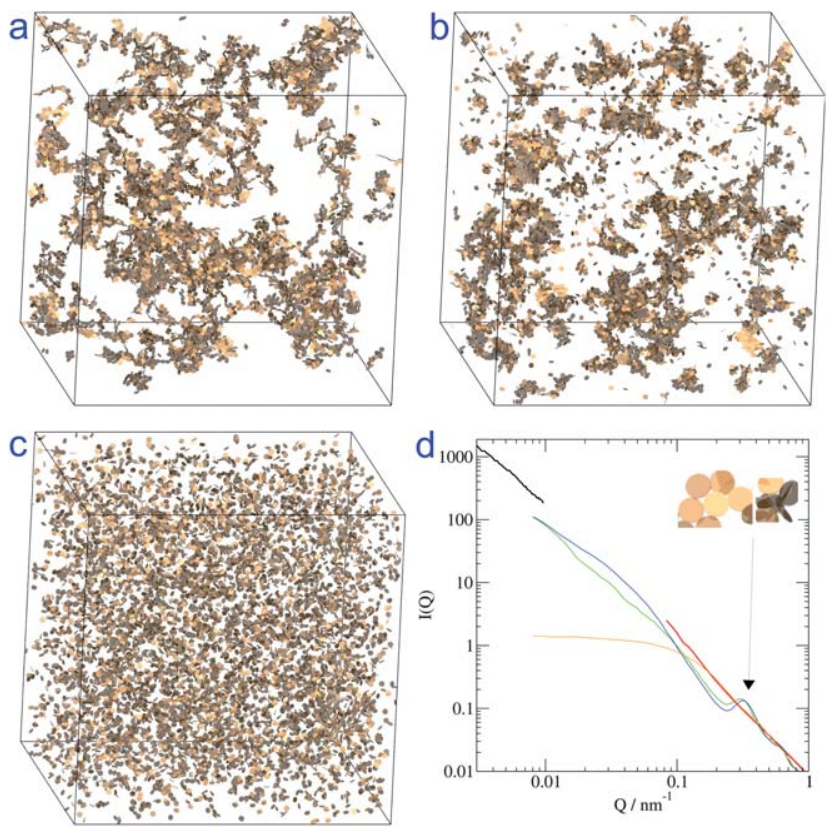

Fig. 5 Typical configurations for the QRPS model at $T^{*}=0.016$ (a), $T^{*}=0.035$ (b) and $T^{*}=0.045$ (c). The corresponding intensity plots are shown in part d $\left(T^{*}=0.016\right.$ - green, $T^{*}=0.035$ - blue, $T^{*}=0.045$ - orange, SAXS - red, SLS - black). 
dispersed platelets as the simulation progresses. A snapshot of a configuration of an arrested network can be seen in Fig. 5a for the QPRS model at $T^{*}=0.016$. The slope of the calculated scattering intensity (see Fig. 5d - green) for the long range correlations (SLS range) matches the slope from the experimental scattering intensity quite well. This suggests that these simulated networks have the correct density and fractal structure. Throughout the text we refer to "slope" as the absolute value of the slope of the scattering intensity curves as they approach the SLS range of $Q$ values. At higher temperatures $\left(T^{*}=0.035\right.$ - Fig. $\left.5 \mathrm{~b}\right)$, closer to the crossover temperature, the long range networks take longer to form, or do not form at all, and instead the particles form clusters that do not percolate the system. These structures produce scattering intensities (see Fig. 5d - blue) that show a slight turn, at long range (small $Q$ ), towards the plateau that is expected for a full dispersion, indicating a dispersion of clusters. We can see that the slope at medium range, $0.1 \mathrm{~nm}^{-1}$, is larger than the experimental one indicating that these clustered structures have a higher density than the ones present in the experimental system.

Looking at short-range correlations (high $Q$, SAXS range) the calculated intensities for systems below the crossover temperature always have a pronounced peak at $\sim 0.3 \mathrm{~nm}^{-1}$ that the experimental one does not show. This peak is related to the local, nearest-neighbour, structure of these clusters and networks. At low temperature the platelets arrange themselves to form small relatively planar structures shown in the left inset of Fig. 5d, that then connect to each other to form the long range structures. At higher temperature the planar structures still dominate the overall structure but another type of local structure starts to emerge. These star shaped structures, as presented on the right inset of Fig. 5d, are composed of three platelets connected by face edge contacts. Both the planar structures and the star ones are related to the electrostatic interaction curves, presented in Fig. 4, for the configurations $\{90,22.5,22.5\}$ and $\{90,22.5,135\}$. It is worth noting that these structures are remarkably different from the typical house of cards ${ }^{18,35-37}$ structure proposed for the gel structure of clays, including Laponite, where pairs of platelets are said to adopt T-shaped structures formed by edge-face interactions. Nevertheless, the results generated with the QPRS model are not in agreement with the experimental ones and hence further hypotheses are tested.

\subsection{Influence of surfactant}

We studied the influence of the surfactant layer by performing simulations with different thicknesses of surfactant and by incorporating an outer attractive layer of surfactant. Some results are presented in Fig. 6 for simulations below the crossover temperature for dispersion. Results for the models with increased surfactant layer thickness (QPIRS) are presented in Fig. 6a $(L=$ $\left.4 \mathrm{~nm}, D=28 \mathrm{~nm}, T^{*}=0.020\right), 6 \mathrm{~b}\left(L=5 \mathrm{~nm}, D=29 \mathrm{~nm}, T^{*}=\right.$ 0.020 ) and $6 \mathrm{~d}$ - green and blue lines, respectively. As in the QPRS model, they also exhibit a correlation peak at $\sim 0.3 \mathrm{~nm}^{-1}$ for short-range correlations. Low temperatures $\left(T^{*}=0.020\right)$ are needed to obtain networked structures for which the slope of the scattering intensity is similar to the experimental one. The structures obtained with this model are either low density networks, based on planar structures (Fig. 6a) and percolating
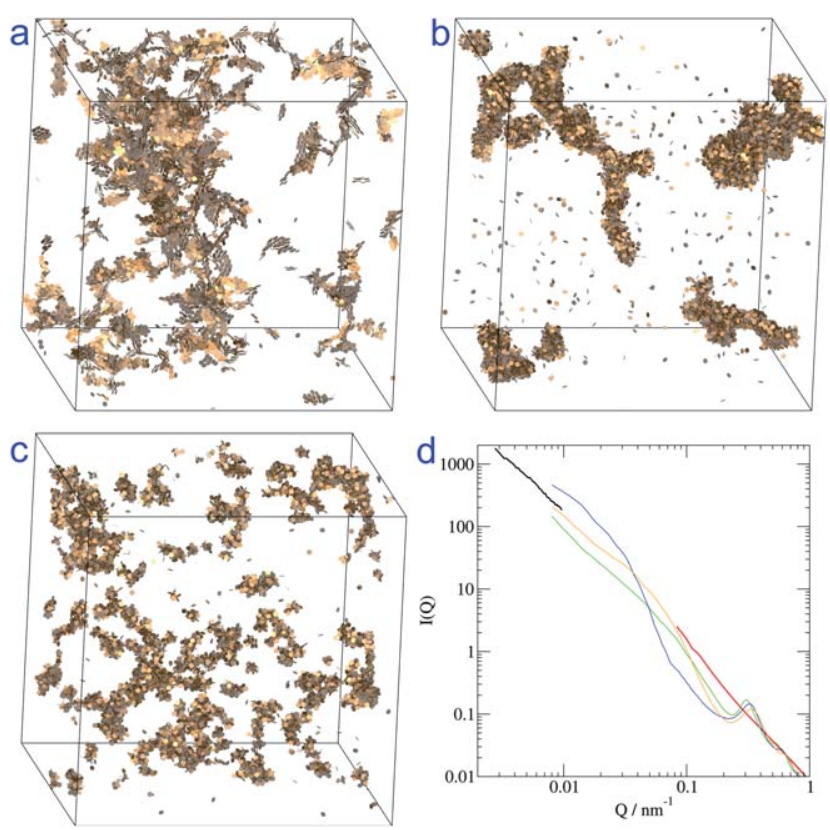

Fig. 6 Typical configurations for the QPIRS model at $T^{*}=0.020$ for different surfactant layers; $1.5 \mathrm{~nm}$ repulsive layer (a), and $2 \mathrm{~nm}$ repulsive layer (b). A typical configuration is shown for the square well attractive surfactant layer model at $T^{*}=0.049$ in part c. The corresponding intensity plots are shown in part $\mathrm{d}(1.5 \mathrm{~nm}$ repulsive - green, $2.0 \mathrm{~nm}$ repulsive - blue, square well attractive - orange, SAXS - red, SLS - black).

the system, or smaller, dense networks (Fig. 6b) based on a mixture of planar and star like structures. The crossover temperature for dispersion, in these models, is lower than for the QPRS model because the electrostatic interactions (Fig. 4) are cut at larger distances and are, therefore, weaker at particle contact. The electrostatic potential energy surface for the QPIRS model displays both weaker and softer interactions with less deep potential wells and lower potential energy barriers between different configurations. Therefore the particles adopt a larger number of short-range configurations giving rise to denser networks than before, obtained closer to the dispersion temperature (see Fig. 6b). In these denser networks a large number of particles form star configurations. The number of contacts for such configurations is larger than for planar structures and so the peak for the short range correlations at $0.3 \mathrm{~nm}^{-1}$ is, consequently, more intense for this model.

For the model with an outer shell of attractive surfactant (QPRSPAS) we obtained some long range networks that generate scattering intensities with approximately the same slope as the experimental one. In Fig. 6c we present a snapshot of a system $\left(L=3 \mathrm{~nm}, D=27 \mathrm{~nm}, L^{\prime}=4 \mathrm{~nm}, D^{\prime}=28 \mathrm{~nm}, U_{S W}=5 \varepsilon T^{*}\right.$, $T^{*}=0.049$, where $U_{S W}$ is the energy of the square-well potential characterising the attraction between surfactant layers) forming such a network and in Fig. 6d (orange line) the respective scattering intensity. However, these networks are formed from very dense structures almost completely built from platelet star-like conformations. Therefore, a very pronounced peak at $\sim 0.3 \mathrm{~nm}^{-1}$ is seen, corresponding to strong short range correlations.

To summarise, we are unable to reproduce the experimental scattering intensity curves using the 'standard' quadrupolar charge distribution of Laponite, regardless of our surfactant 
model. These results show that the main problem in modelling Laponite gels is not in building a model that generates long range structures with the correct fractal dimension, but in designing a model that generates local arrangements of platelets with the correct short-range scattering intensity.

\subsection{Model with quadrupole and dipole plus repulsive surfactant}

Simulations with the model incorporating a quadrupole and a dipole (QDPRS) were performed to test the influence of a hypothetical small dipole placed along the equatorial plane of Laponite platelets. The dipole strength was varied by changing the charge magnitude and keeping the distance between charges constant. As we will see, the complexity of these platelet interactions, introduced by the presence of the dipole, is enough to generate a much wider range of structures, some of them presenting a very good match with our experimental results. In Fig. 7, we present the most relevant results, corresponding to different conditions of temperature and dipole strength, that we obtained for this model.
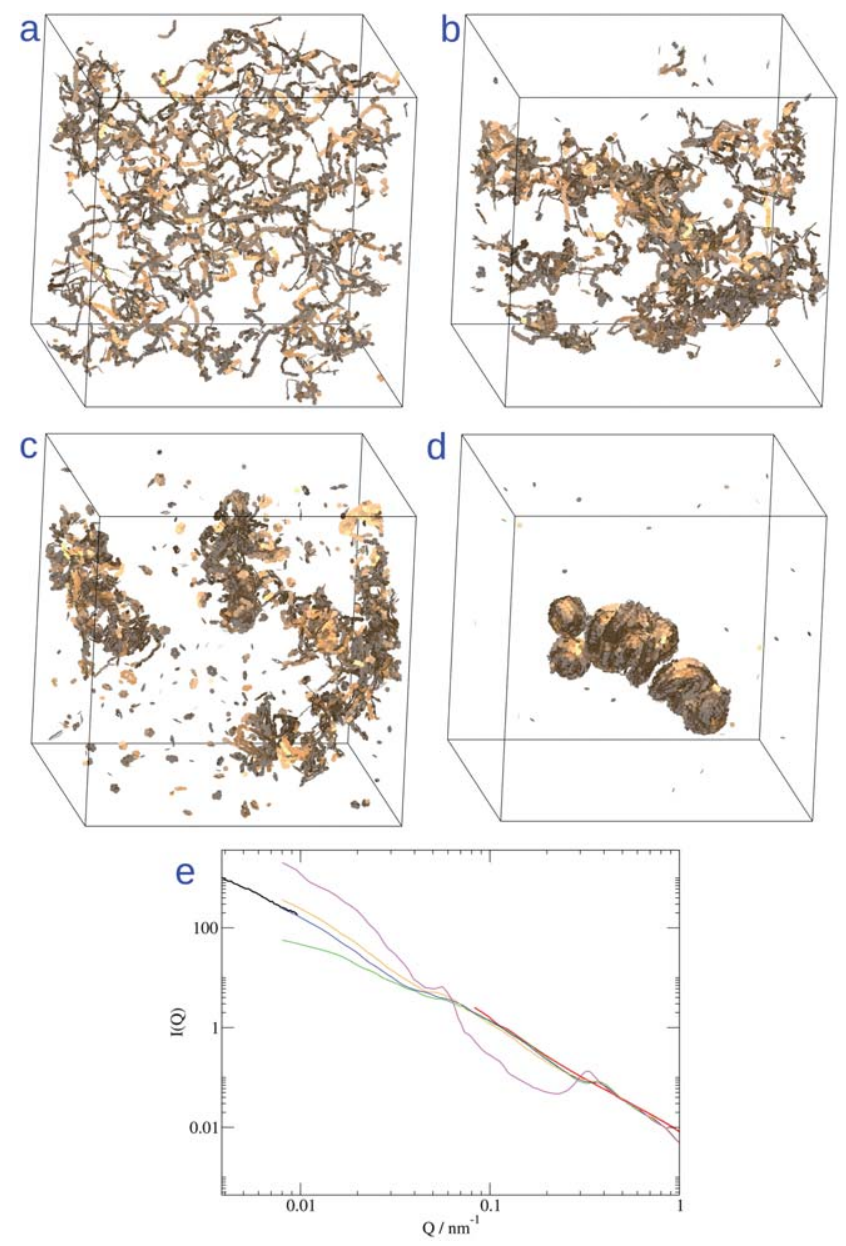

Fig. 7 Typical configurations for the QDPRS model with $q=2.0 e$ at $T^{*}=0.020$ (a), $q=2.0 e$ at $T^{*}=0.035$ (b) and $q=2.0 e$ at 0.053 (c), and $q=1.5 e$ at 0.045 (d). The corresponding intensity plots are shown in part e $(q=2.0 e$ at 0.020 - green, $q=2.0 e$ at 0.035 - blue, $q=2.0 e$ at 0.053 orange, $q=1.5 e$ at 0.045 - magenta, SAXS - red, SLS - black).
The quadrupolar-dipolar disc model, bearing charge distributions (as opposed to point quadrupole and dipole moments) is incredibly rich in the morphology of the structures it can yield and just as an example we show, in Fig. 7d, the spontaneous self assembly of globular structures from a dispersion of platelets with $L=3 \mathrm{~nm}, D=27 \mathrm{~nm}$ and $q=1.5 e$. These structures bear some resemblance to self-assembled capsids reported recently ${ }^{38}$ constructed from 'designer' molecules, and also to capsids constructed via directed assembly on a spherical template. ${ }^{39}$ The scattering intensity of these structures is presented in Fig. 7e (magenta line) showing a high slope in the SLS range, corresponding to the higher density of these structures, and a strong peak in the SAXS region due to the strong close range correlations in these structures. From the snapshot we can see that the walls of these structures are formed by chains of platelets placed side by side in order to form a curved wall. The structures are not completely empty but the interior definitely has a lower density than the walls. This result points to a potential route for the experimental production of shell like structures from suspensions of discotic particles, although the relationship between quadrupole, dipole and geometric parameters (e.g. aspect ratio) that gives rise to this phenomena needs further investigation. These structures, if they are found to actually occur in aqueous solutions, might also be relevant for the formation of life on Earth, considering that clay (Montmorillonite) surfaces are thought to catalyse the formation of vesicles. ${ }^{40,41}$

The case with $L=3 \mathrm{~nm}, D=27 \mathrm{~nm}, q=2 e$ (see Fig. 7a, b and $\mathrm{c}$ and Fig. $7 \mathrm{e}$ - green, orange and blue lines) is the one that best matches the experimental results. Analysing the calculated scattering intensities, shown in Fig. 7e, it is clear that the peak corresponding to the short range correlations, at $\sim 0.3 \mathrm{~nm}^{-1}$, is very weak. This means that the short range arrangements of the platelets must be very different from before. Indeed, from the snapshots of Fig. 7, we can see that the particles are now arranged into chains, rather than plates or star-like structures, having a lower number of contact neighbours. These chains are a consequence of the alignment of the dipoles in the platelets, although the effect of the quadrupolar charge distribution is still very important. The quadrupolar repulsive interaction for configurations where the platelets approach face-to-face prevents the platelets from stacking into small columns and, therefore, forces the platelets to form open structures. At low temperature (see Fig. 7a for $T^{*}=0.020$ ) the system forms very low density networked structures, that percolate the whole simulation box. This 'string'-like or 'polymer' phase is to be expected for a strongly dipolar dispersion. Actually, this structure appears to closely resemble the structures seen below the percolation threshold for dipolar dumbbells. ${ }^{42}$ The slope of the corresponding calculated scattering intensity (see Fig. 7e - green) in the SLS range is $-0.95 \pm 0.03$, in agreement with what is expected for single chains $(-1)$. Nevertheless, this is clearly lower than the experimental one, meaning that these networks are "too open" when compared to the structure of the experimental system. From the simulations we observed that the platelets in the chains were very static and we correlate this observation with the presence of a small peak, at $\sim 0.3 \mathrm{~nm}^{-1}$, corresponding to short range correlations. At higher temperatures we obtained better agreement with the experimental results since, due to higher mobility of the platelets, the peak for short range correlations 
becomes increasingly flattened. A snapshot of the network that gave the best match between the calculated and experimental scattering intensities is presented in Fig. $7 \mathrm{~b}\left(T^{*}=0.035\right)$ and the respective intensity in Fig. 7e (blue line). This network is composed mainly of platelet chains but now, in contrast with the very open chains at low temperature, they can form some medium range planar aggregates in which two or three chains pair up, side by side. At higher temperatures the mobility of the particles is highly increased and the networks start to collapse into more dense structures, as presented in Fig. $7 \mathrm{c}\left(T^{*}=0.053\right)$ that stay in equilibrium with oligomeric units which are mostly planar or ring shaped. Due to the higher density these networks have higher fractal dimension as indicated by the slightly larger slope of the calculated scattering intensity (see Fig. 7e - orange line). The behaviour observed in the simulations seems to indicate that a rise in temperature, below the crossover temperature for dispersion, may speed up the formation of higher density structures and therefore increase the rate of sedimentation of the samples. This effect remains to be confirmed as higher temperature experiments with styrene are, at the moment, too complicated to perform. There is still the matter of the small peak present in the calculated scattering intensity at short range that is not present in the experimental scattering intensity. We suggest that this small peak might be a consequence of the monodisperse simulation model. Laponite particles, however, have a range of diameters (thickness is fairly constant) and face shape anisotropies $^{11}$ which could result in the smooth scattering intensity seen in the experiments.

\section{Summary}

By fine-tuning a simulation model we are able to closely match the scattering intensities generated by large-scale Monte-Carlo simulations and experiments for a particular surface modified Laponite dispersed in styrene. Our results suggest that Laponite might have a permanent electrostatic dipole in the plane of the crystal. This possibility seems not to have been mentioned in the literature before, which brings into question some of the early theoretical work on polymer-clay phase behavior. ${ }^{43-45}$ In that work concerning clay particles dispersed in a polymer melt clay particles are represented in terms of hard disks modeled with excluded volume interactions only, ${ }^{43}$ or by hard disks with an anisotropic interaction that favours parallel alignment of disks. ${ }^{44,45}$ Our work suggests these models are likely to be inadequate for theoretical treatment of both monomer-Laponite and polymer melt-Laponite mixtures. Also note that several experimental reports provide hints that inclusion of an in-plane electrostatic dipole should be considered. For example, in electrorheological experimental studies on suspensions of organically modified Laponite in non polar solvents ${ }^{46,47}$ the authors refer to dipole moments induced by an electric field. We suggest, instead, that Laponite carries a permanent dipole that is enhanced in the presence of an electric field by the polarization of surfactant layers, and therefore produces the strong alignment of the platelets observed in these studies. Also consider the cryofracture imaging experiments of Mourchid et al. ${ }^{17}$ for Laponite in brine (without any electric field) that appear to show Laponite particles forming kinds of broken chain-like structures at higher concentrations. In their images the Laponite particles are well separated, as expected for Laponite in brine, but nevertheless they appear to be aligned into short chains, potentially indicative of an in-plane dipole moment.

However, we cannot state definitively that this is the only simulation model able to match the experimental results; it is simply the only model that we tried that was able. Nevertheless, it does seem that whatever simulation model is designed, its interactions should lead to a low number of platelet contacts, since otherwise a sharp peak in the SAXS range will be observed. It follows that for our system of Laponite in styrene we can rule out house-of-cards ${ }^{36}$ or similar structures. Indeed, the best match with experiment is generated by a networked or clustered system that more closely resembles the 'cellular foam' structure proposed by Morris and $\mathrm{Zbik}^{48}$ for smectite in water, and the 'bifurcated' string-phase seen in simulations of dipolar dumbbells. ${ }^{42}$ Indeed, the apparent agreement between our simulated structure in Fig. 6b and a TEM image of a dried cluster of surface modified Laponite $^{7}$ is remarkable. However, the range of morphologies exhibited by our quadrupolar-dipolar platelet model is rather wide, including intriguing self-assembled globular structures, and it seems likely to us that this could be mirrored by experimental systems for a range of clay systems dispersed in a range of solvents over a range of temperatures. The classification of such gels into 'strong' and 'weak' kinds following Pluart et al., ${ }^{9}$ while suitable for some systems, might well be too simplistic for general use.

Ultimately, this work suggests that it will be very difficult to obtain good isotropic dispersions of Laponite, and therefore perhaps other clays, without any clustering in typical monomeric solvents such as styrene. Our results suggests these systems are dominated by a combination of dipolar and quadrupolar interactions, and since dipole - dipole interactions are relatively longranged it could be difficult to eliminate their effects unless very thick layers of surfactant can be achieved. This could be a major problem for the development of polymer-clay nanocomposites formed via the in situ polymerisation route and the development of polymer-clay nanocomposites with well controlled structures. While we cannot be sure whether or not gelled structures occurred in earlier work involving Laponite that motivated this work, ${ }^{7,8}$ our results here do question the existence of isotropic dispersions without clustering in those systems.

Finally, we remark on the utility and novelty of these kinds of platelet-level simulations for understanding clay dispersions. By matching, for the first time, large-scale simulation data with experimental scattering data over multiple length-scales for a clay dispersion, we are able to gain new insight into dispersion structure, and therefore into effective interactions between clay particles, that is difficult to obtain using only simulations or experiments alone or indeed any method that analyses only relatively limited structural length-scales. Although the application here is to a synthetic clay dispersion, we expect our methods can be applied to a wide range of platelet dispersions, synthetic or otherwise.

\section{References}

1 B. Chen, J. R. G. Evans, H. C. Greenwell, P. Boulet, P. V. Coveney, A. A. Bowden and A. Whiting, Chem. Soc. Rev., 2008, 37, 568-594.

2 F. Hussain, M. Hojjati, M. Okamoto and R. E. Gorga, J. Compos. Mater., 2006, 40(17), 1511-1575. 
3 T. D. Fornes, P. J. Yoon, D. L. Hunter, H. Keskkula and D. R. Paul, Polymer, 2002, 43(22), 5915-5933.

4 D. Burgentzle, J. Duchet, J. F. Gerard, A. Jupin and B. Fillon, J. Colloid Interface Sci., 2004, 278(1), 26-39.

5 Y. S. Choi, H. T. Ham and I. J. Chung, Chem. Mater., 2004, 16(13), 2522-2529.

6 M. Yoonessi, H. Toghiani, W. L. Kingery, and C. U. Pittman, Macromolecules, April, 2004, 37(7), pp. 2511-2518.

7 E. S. H. Leach, A. Hopkinson, K. Franklin and J. van Duijneveldt, Langmuir, 2005, 21(9), 3821-3830.

8 M. McAlpine, N. E. Hudson, J. J. Liggat, R. A. Pethrick, D. Pugh and I. Rhoney, J. Appl. Polym. Sci., 2006, 99(5), 2614-2626.

9 L. Le Pluart, J. Duchet, H. Sautereau, P. Halley and J. F. Gerard, Appl. Clay Sci., 2004, 25(3-4), 207-219.

10 J. Li, J. M. Fitz-Gerald and J. P. Oberhauser, Appl. Phys. A: Mater. Sci. Process., 2007, 87(1), 97-102.

11 E. Balnois, S. Durand-Vidal and P. Levitz, Langmuir, 2003, 19(17), 6633-6637.

12 R. A. Vaia, W. D. Liu and H. Koerner, J. Polym. Sci., Part B: Polym. Phys., 2003, 41(24), 3214-3236.

13 P. Sandkuhler, J. Sefcik and M. Morbidelli, Langmuir, 2005, 21(5), 2062-2077.

14 M. Dijkstra, R. van Roij and R. Evans, J. Chem. Phys., 2000, 113(11), 4799-4807.

15 J. A. C. Veerman and D. Frenkel, Phys. Rev. A: At., Mol., Opt. Phys., 1992, 45(8), 5632.

16 R. P. S. Fartaria and M. B. Sweatman, Chem. Phys. Lett., 2009, 478 (4-6), 150-154.

17 A. Mourchid, A. Delville, J. Lambard, E. Lecolier and P. Levitz, Langmuir, 1995, 11(6), 1942-1950.

18 M. Dijkstra, J. P. Hansen and P. A. Madden, Phys. Rev. E: Stat. Phys., Plasmas, Fluids, Relat. Interdiscip. Top., 1997, 55(3), 3044-3053.

19 M. Kroon, W. L. Vos and G. H. Wegdam, Phys. Rev. E: Stat. Phys., Plasmas, Fluids, Relat. Interdiscip. Top., 1998, 57(2), 1962.

20 J. O. Fossum, Phys. A, 1999, 270(1-2), 270-277.

21 D. G. Rowan, J. P. Hansen and E. Trizac, Mol. Phys., 2000, 98(18), 1369-1378.

22 R. J. F. L. de Carvalho and N. T. Skipper, J. Chem. Phys., 2001, 114 (8), 3727-3733.

23 Y. Aray, M. Marquez, J. Rodriguez, S. Coll, Y. Simon-Manso, C. Gonzalez and D. A. Weitz, J. Phys. Chem. B, 2003, 107(34), 8946-8952.

24 S. Kutter, J. P. Hansen, M. Sprik and E. Boek, J. Chem. Phys., 2000, 112(1), 311-322.

25 A. Delville and P. Levitz, J. Phys. Chem. B, 2001, 105(3), 663-667.
26 S. Mossa, C. De Michele and F. Sciortino, J. Chem. Phys., 2007, 126 (1), 014905.

27 B. Jonsson, C. Labbez and B. Cabane, Langmuir, 2008, 24(20), $11406-11413$.

28 A. R. Edmonds, Angular Momentum in Quantum Mechanics, Princeton University Press, 1957.

29 W. H. Press, S. A. Teukolsky, W. T. Vetterling, and B. P. Flannery, Numerical Recipes in Fortran 77, Cambridge University Press, 2nd ed., 2001.

30 G. Odriozola, M. Romero-Bastida and F. D. Guevara-Rodriguez, Phys. Rev. E: Stat., Nonlinear, Soft Matter Phys., 2004, 70(2).

31 W. B. Russel, D. A. Saville, and W. R. Schowalter, Colloidal Dispersions, Cambridge University Press, 1998.

32 D. Frenkel and B. Smit, Understanding Molecular Simulations: From Algorithms to Applications, Academic Press, 2nd ed., 2002.

33 L. Harnau, Mol. Phys., 2008, 106(16-18), 1975.

34 J. P. Hansen and I. R. McDonald, Theory of Simple Liquids, Elsevier Ltd., London, UK, 2nd ed., 1986.

35 M. Dijkstra, J. P. Hansen and P. A. Madden, Phys. Rev. Lett., 1995, 75(11), 2236.

36 P. F. Luckham and S. Rossi, Adv. Colloid Interface Sci., 1999, 82(1-3), 43-92.

37 P. Mongondry, J. F. Tassin and T. Nicolai, J. Colloid Interface Sci., 2005, 283(2), 397-405.

38 D. C. Rapaport, J. Phys.: Condens. Matter, 2010, 22(10), 104115.

39 E. Mani, E. Sanz, P. G. Bolhuis and W. K. Kegel, J. Phys. Chem. C, 2010, 114(17), 7780-7786.

40 M. M. Hanczyc, S. M. Fujikawa and J. W. Szostak, Science, 2003, 302 (5645), 618-622.

41 M. M. Hanczyc, S. S. Mansy and J. W. Szostak, Origins Life Evol. Biosphere, 2006, 37(1), 67-82.

42 M. A. Miller, R. Blaak, C. Lumb and J. P. Hansen, J. Chem. Phys., 2009, 130(11), 114507.

43 Y. Lyatskaya and A. C. Balazs, Macromolecules, September, 1998, 31 (19), pp. 6676-6680.

44 V. V. Ginzburg and A. C. Balazs, Macromolecules, August, 1999, 32 (17), pp. 5681-5688.

45 A. C. Balazs, V. Ginzburg, and Y. Lyatskaya, Polymer-Clay Nanocomposites, Wiley, 2000.

46 K. P. S. Parmar, Y. Meheust, B. Schjelderupsen and J. O. Fossum, Langmuir, 2008, 24(5), 1814-1822.

47 B. X. Wang, M. Zhou, Z. Rozynek and J. O. Fossum, J. Mater. Chem., 2009, 19(13), 1816-1828.

48 G. E. Morris and M. S. Zbik, Int. J. Miner. Process., 2009, 93(1), 20 25. 